\title{
Analysis for bio-inspired thrown-box assisted message dissemination in delay tolerant networks
}

\author{
Bo Gu • Xiaoyan Hong • Pu Wang
}

Published online: 3 August 2011

(C) Springer Science+Business Media, LLC 2011

\begin{abstract}
Inspired by biological communication, the strategy of deploying communication and storage equipment called thrown box is proposed to increase message delivery probability and to reduce transmission latency in delay tolerant networks. In this paper, we study the thrown-box assisted message dissemination models by analyzing a few cases on the message delivery rate and related latency distribution. We divide such communications into two processes. We first model the message delivering process among thrown boxes and derive time related message distribution on the boxes. Then we investigate the message collection process to obtain the expected number of informed collectors as a function of time. In addition, we analyze the latency distribution for message collection. Our analysis is derived based on a discrete Markov Chain model. The numerical examples are provided to validate our model and to examine the features of message dissemination under different network scenarios. The factors such as message relay and lifetime are considered. The results show that the tradeoff exists between the number of the boxes and the message lifetime, etc. In summary, our results will help storage management and delay management in DTNs and provide guidelines for applications of search and surveillance.
\end{abstract}

Keywords Bio-inspired communications · Delay tolerant networks · Delay tolerant network routing · Performance

This work is supported in part by NSF Awards No. 0829827.

\footnotetext{
B. Gu $\cdot$ X. Hong $(\bowtie)$

Department of Computer Science, University of Alabama, Tuscaloosa, AL 35487, USA

e-mail: hxy@cs.ua.edu

P. Wang

Department of Mathematics, University of Alabama, Tuscaloosa, AL 35487, USA
}

analysis $\cdot$ Heterogeneous mobile and static sensor networks · Latency analysis

\section{Introduction}

Insects, fish, birds, etc have inspired and attracted much attention in many engineering fields, including computing, networking and distributed systems. For example, swarm intelligence, a model based on ants, has attracted extensive studies for communication in self-organized networks $[7,8,11,17,25]$. At a more intelligent level, primate species can provide biological inspirations for the design and implementation of cooperative communications and networking protocols. Primates have higher cognitive capabilities, enhanced learning, multiple communication modes and a variety of individual capabilities that must be integrated with social and group life $[2,5,9,10]$. Taking apes as example, they use vocal calls, facial expression and body language for communication directly and explicitly when they are within a certain range of distance. However, to pass information to peers not in vicinity, they leave trails such as footprint or pheromone at special locations to guide or warn the relevant apes. By retrieving the delicate pheromone from the trails, the latter apes are able to act accordingly. The functions of trail leaving in primates $[16,22]$ include territorial demarcation, ownership of resources, mate attraction and the establishment of social role.

On the other hand, intermittent communication connections between mobile wireless nodes can be observed in many network scenarios, such as wildlife tracking, surveillance, robotic search, emergency rescue, etc. Message dissemination in above networks for non-real time tasks and missions is achieved by using store-carry-and-forward methods based on encountering opportunities because the 
instant end-to-end paths may not exist when mobile nodes initialize communications. In this area, named as delay tolerant networks (DTNs), a variety of methods are proposed to achieve message dissemination which include estimating a better forwarder to carry a message or utilizing several copies of a message to speed up the delivery. Inspired by the observation that the primates select locations and leave scents to pass information, some recent works have used the idea of deploying stationary devices to act as pheromone at specific locations [29]. The stationary devices (or thrown boxes) can be small and cheap devices with wireless antennas and limited memory. They can be deployed quickly for the role of message relay. Issues of enhancing the capacity and optimizing the deployment of these boxes are studied in $[28,29]$.

In addition, often there are application scenarios, such as search and surveillance of complex city areas, where the number of mobile devices is a resource constraint for network communications, but applications anticipate quick message disseminations even though this is a delay tolerant networks. In these applications, latency analysis based on the thrown box assisted dissemination model is essential guidelines for the success of the application missions. Further investigations are largely needed. Inspired by the apes (as information initiators) trying to increase the contact probability of the message receivers by leaving pheromone at possible areas, we introduce a discrete model to describe such a communication method and analyze the related communication metrics. Here, considering that the pheromone usually lasts for a certain period of time before disappearing, we assign the messages left at the thrown boxes to bear a lifetime. While this time period depends on the locations and intensities of the pheromone left by the apes, here it may depend on the popularity of the boxes, buffer sizes and message themselves. A receiver should be able to retrieve a message before it expires.

This paper analyzes a few communication relay models which differ in the actions of the sender, relay nodes and the receivers when they receive messages from thrown boxes. The analysis aims at answering the question regarding to retrieving a message from the thrown box for the first time by a receiver. The message lifetime and distributions at thrown boxes are all the factors considered for the message collection. The movement pattern of node in the analytical model capitalizes on a landmark based model where all the mobile nodes travel among landmarks and exchange messages through the thrown boxes deployed at these landmarks. After that we use a two-processes approach in the work, namely, we first model the message delivering process among thrown boxes and derive the time evolution about the box having message; then, we investigate the process of message collection. The results of the two processes are used to obtain the expected number of informed collectors varying with time. In addition, we analyze the latency distribution for message collection. Our analysis is derived based on a discrete Markov Chain model. The numerical examples are provided to validate our model and to examine the features of message dissemination under different network scenarios. The results show that the longer lifetime will lead to shorter latency. There are also relationships between thrown-box location distributions, message delivery paths and the latency. In summary, our results will help store management and delay management in DTNs.

In the following sections, we first discuss the related works in Sect. 2. Based on the thrown box assisted pattern, Sect. 3 provides an introduction of the network model and dissemination model. The analytical results about scattering message at thrown boxes are given in Sect. 4. Adding the relay function of thrown boxes, the process of message collection is introduced in Sect. 5. Further, Sect. 6 gives latency study of message dissemination to the mobile devices. We give the numerical results and conclude the paper in Sect. 7 and Sect. 8 respectively.

\section{Related work}

Many related works focus on the efficient routing protocol in DTN. By using a network graph with time-varying capacity and propagation delay, Jain et al. [14] propose a framework of routing to minimize the delay of message delivery. The works in [1] treat the message routing as a resource allocation problem in which each packet in the buffer of node is decided to be replicated or not in order to optimize a specific routing metric. A node with special network structural properties can also be regarded as active node for forwarding message. In $[6,13]$, the social network related methods are used to identify the nodes with high betweenness centrality which measures the importance of node on the communication paths between other nodes. Island Hopping [18] is another efficient mobility-assisted routing protocol that relies on the cluster based mobility model. Mobile message ferry approach for routing in mobile ad hoc network is discussed in [28]. The two types of scheme: Node-Initialed scheme and Message Ferry-Initiated MF scheme are proposed to improve data delivery and reduce energy assumption. The strategy in [29] makes use of some stationary devices deployed between mobile nodes to facilitate message exchange. Three different scenarios are considered to derive the optimal locations for deployment, which are contact and traffic based case, contact based case and oblivious model. Finally the optimal deployment is formulated as a mixed integer programming problem and solved by the greedy algorithm.

Bio-inspired approaches have been used in many ways in communications. Swarm intelligence [3] is used to describe 
the collective behaviors in biological system such as ant colonies, bird flocking, animal herding, bacterial growth and fish schooling. The works inspired by swarm intelligence are proposed in $[4,19,20]$ to derive robust and adaptive communications. In [4], the authors introduce a distributed and mobile agents based routing with superior performance inspired by the cooperation in ant colony. In [20], an efficient multi-cast routing is proposed by constructing the minimum forwarding set, which is motivated by the pheromone based path selection in biological system. Besides that, the concept of pheromone which well measures the attraction and repulsion among animals is applied to implement a novel clustering algorithm [19]. In addition, Levy walk is a mobility pattern that reflects the forage behavior. By employing Levy walk, the search for sparsely and randomly distributed targets can be the optimal [26]. In DTN, Levy message ferries are proposed in [21].

The modeling and analysis works are usually performed for protocol design and evaluation. In the paper [12], the authors make use of a semi-Markov chain to analyze the contact probability of two nodes at equilibrium and also the probability that one node meets another at a specific location within certain time period. Spray and Wait protocol [24] derives the relationship between the number of nodes and the amount of copies in order to get the optimal number of copies to spread. By using the two-state Markov model and geometric distribution, the expected hitting time (to a location) and meeting time (of two nodes) are found for time-variant Community mobility model in [27]. The authors in [23] analyze the hitting time or meeting time for random direction and random way-point models by providing a closed forms and show that these results can be used to analyze the performance of mobility-assisted routing schemes. A similar analytical work proposed in [30] studies the encounter probability of two nodes meeting each other and the time it takes for two nodes to meet.

Since the thrown box is introduced to enhance communication in DTN, our analysis model is different from previous works in terms of the relay effect from the thrown box and the landmark based mobility model. Besides the message dissemination among boxes and message collection process, the message expiration at box is considered as well. Thereby, the analytical results derived from our model could help to understand the thrown box based message dissemination, also benefit the box deployment for efficient communication.

\section{Message dissemination model}

Inspired by the observation that the primates select locations and leave scents to pass information, communications

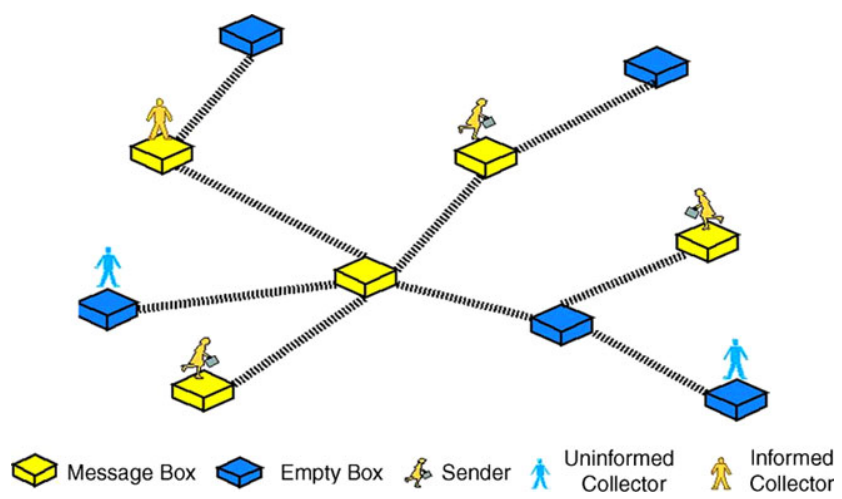

Fig. 1 Scenario of message dissemination

in DTN can use the "thrown-boxes", i.e., static communication and storage devices at selected locations, to store messages and also to create opportunities for other nodes to pickup those messages, and maybe to carry them to other locations. Typically, the locations of the thrown boxes can be the places where the mobile senders and collectors visit frequently. Messages are temporally stored at the boxes when they are visited by the sender nodes, waiting to be collected by destination nodes or relay nodes before expiration. Figure 1 gives a sample scenario of this communication model. Three entities exist in the network which are message sender, message collector and thrown box. In our model, there can be direct message exchange between the sender and the receiver when encountering at the same box, or the receiver may find message by checking the visiting box. In our scenario, the box having message stored is called message box and empty box denotes the box without message. In the following analysis we differentiate cases of whether the message can be relayed or not, whether the message has a lifetime or not. These factors will affect the distributions of the message at the boxes and the message collection rate (i.e., delivery rate) and latency.

Assume there are total $N_{s}+N_{c}$ mobile nodes in network among which $N_{s}$ nodes play the role of information initiators and the rest $N_{c}$ nodes are viewed as the message collectors. In addition, there are total $B$ thrown boxes where the mobile nodes can frequently visit and stay for a certain time period. Suppose all the message senders have the same message copies and no transmissions happen during the transition between boxes. A matrix with $B \times B$ entries that reflect the topology of network and visiting preference of box is used to express the mobility. Each entry $e(i, j)$ denotes the probability that the mobile node starts from box $i$ to box $j$ in next time step. In addition, we assume all mobile nodes move independently and the dissemination process is based on discrete time. The transition time between boxes is ignored due to the assumption that dwelling time at box is much longer than the time in motion. 
Table 1 Table of symbols

\begin{tabular}{ll}
\hline$B$ & Number of boxes \\
$N_{s}$ & Number of sender nodes \\
$N_{c}$ & Number of collector nodes \\
$N$ & Number of nodes, $N=N_{c}+N_{s}$ \\
$p_{i, t}$ & Probability that collector node $i$ has the message at time $t$ \\
$e$ & Probability of message expiration at box \\
$\alpha_{b, t}$ & Probability that box $b$ has the message at time $t$ \\
$\beta_{b, t}$ & Probability that box $b$ does not receive the message \\
& from any nodes at time $t$ \\
$M_{i}$ & Transition Matrix of sender node $i$ with $B \times B$ entries \\
$m_{(i, b), t}$ & $\begin{array}{l}\text { Probability that sender node } i \text { reaches box } b \text { at time } t \\
Q_{i}\end{array}$ \\
$q_{(i, b), t}$ & Transition Matrix of collector node $i$ with $B \times B$ entries \\
$\lambda_{t}$ & Probability that collector node $i$ reaches box $b$ at time $t$ \\
$\mu_{t}$ & Expect number of boxes having message at time $t$ \\
$h_{i, t}$ & Expect number of collectors having message at time $t$ \\
& Probability that collector node $i$ receives \\
$\eta_{t}$ & message at time $t$ for the first time \\
& Expect number of collectors having message at time $t$ \\
\hline
\end{tabular}

In general, we divide the message dissemination through thrown boxes into two phases. Firstly, we only focus on the scenario with message sender and thrown box, where the mobile message senders drop messages at every visited box. Since the message stored at each box has an expiration probability, it is necessary to model the message scatter at box and study the time evolution of message box. This analysis of message dissemination among boxes is given in Sect. 4 . In the second phase discussed in Sect. 5, the message collection process is considered where the mobile collector can retrieve data by searching in each visiting box. Through modeling the message reception during each time step, we can derive the probability of collection at time $t$ and estimate the expected number of informed collectors. All the symbols shown in the model are given in Table 1.

\section{Analysis of message dissemination among boxes}

We first study the process of message dropping at boxes in order to obtain the probability of box having message and predict its expected amount. The message dissemination among boxes depends on mobility of message sender, message expiration and the previous state of box. Specifically, when a message initiator arrives at an empty box, then a copy of message is temporally stored at that box. At next time step, the message could be expired and that box is empty again. We denote by $e$ the probability of message expiration at box. When the probability $e$ is zero, the message left by the sender always exists at box. In another extreme case when $e=1$, the message left at the box by sender keeps valid only for one time step. In other words, the message exchange has to rely on the node contact at the same box.

Both (1) and (2) are proposed to describe the process of message dissemination among boxes. In (1), the probability $\beta_{b, t}$ that box $b$ does not receive the message from any nodes at time $t$ equals to the probabilities that all the message senders don't reach box $b$ at time $t$. Equation (2) gives the probability $1-\alpha_{b, t}$ that box $b$ has no message at time $t$, where the first term on the right side of equation denotes the probability that this box has no data at time $t-1$ and is still unreached by any sender at $t$ and the second term represents the likelihood that message is received at time $t-1$ but expired at time $t$.

$\beta_{b, t}=\prod_{j=1}^{N_{s}}\left(1-m_{(j, b), t}\right)$,

$1-\alpha_{b, t}=\left(1-\alpha_{b, t-1}\right) \beta_{b, t}+e \alpha_{b, t-1}$

Based on above equations, the expected number of message boxes until time $t$ is $\lambda_{t}=\sum_{b=1}^{B} \alpha_{b, t}$.

Theorem 1 Given the limiting probability $m_{(j, b)}$ of any sender $j$ and message expiration probability e. For large $t$,

$\lambda_{t}=\sum_{b=1}^{B} \frac{1-\prod_{j=1}^{N_{s}}\left(1-m_{(j, b)}\right)}{1-\prod_{j=1}^{N_{s}}\left(1-m_{(j, b)}\right)+e}$

Proof By rearranging (2), we can get

$\alpha_{b, t}=1-\beta_{b, t}+\left(\beta_{b, t}-e\right) \alpha_{b, t-1}$

Iteratively solve the equation, then

$$
\begin{aligned}
\alpha_{b, t}= & 1-\beta_{b, t}+\left(\beta_{b, t}-e\right) \alpha_{b, t-1} \\
= & 1-\beta_{b, t}+\left(\beta_{b, t}-e\right)\left(1-\beta_{b, t-1}\right) \\
& +\left(\beta_{b, t}-e\right)\left(\beta_{b, t-1}-e\right) \alpha_{b, t-2} \\
= & \cdots \\
= & 1-\beta_{b, t}+\sum_{j=0}^{t}\left(\prod_{i=j+1}^{t}\left(\beta_{b, i}-e\right)\right)\left(1-\beta_{b, j}\right) \\
& +\prod_{i=1}^{t}\left(\beta_{b, i}-e\right) \alpha_{b, 0}
\end{aligned}
$$

For large $t$, we let $\beta_{b}$ to be the limiting probability of $\beta_{b, t}$ and the term $\prod_{i=1}^{t}\left(\beta_{b, i}-e\right) \alpha_{b, 0}$ approaches 0 . Then $\alpha_{b}$ is 
introduced to express the limiting probability for large $t$,

$\alpha_{b}=\frac{1-\beta_{b}}{1-\beta_{b}+e}=\frac{1-\prod_{j=1}^{N_{s}}\left(1-m_{(j, b)}\right)}{1-\prod_{j=1}^{N_{s}}\left(1-m_{(j, b)}\right)+e}$

Thus, $\lambda_{t}=\sum_{b=1}^{B} \frac{1-\prod_{j=1}^{N_{s}}\left(1-m_{(j, b)}\right)}{1-\prod_{j=1}^{N_{S}}\left(1-m_{(j, b)}\right)+e}$.

From the result in Theorem 1, we know that all the boxes will finally hold the data when the message does not expire. In other words, if $e$ equals to $0, \lambda_{t}$ will reach to $B$. However, if $e>0$, there will be only a part of the boxes having valid data and the amount is limited by $\lambda_{t}$. In addition, the mobility of message sender can decide whether a box has the message or not. For example, if the probability that all the senders visit box $b$ is zero, then $1-\prod_{j=1}^{N_{s}}\left(1-m_{(j, b)}\right)$ is zero and further $\alpha_{b, t}=0$. In such case, it means the box $b$ will never receive any message from sender.

\section{Analysis of message collection}

In this section, the message collectors bear a part in the data dissemination what will play the role of message dissemination. Two different types of collector are discussed in this process. The first type is called independent collector. Such collectors receive message independently and do not relay the received message to other uninformed collector. The second type is cooperative collector which can continuously forward the message. Specifically, once a cooperative collector is informed by picking up message at certain box, it will behave like sender and drop message at the next visiting box for other uninformed collector.

\section{Independent collectors}

For any independent collector $i$, the model that describes the probability of message reception at time $t$ is expressed by the following equations.

$$
\begin{aligned}
& \beta_{b, t}=\prod_{j=1}^{N_{s}}\left(1-m_{(j, b), t}\right), \\
& 1-\alpha_{b, t}=\left(1-\alpha_{b, t-1}\right) \beta_{b, t}+e \alpha_{b, t-1}, \\
& 1-p_{i, t}=\left(1-p_{i, t-1}\right) \prod_{b=1}^{B}\left(1-\alpha_{b, t} q_{(i, b), t}\right)
\end{aligned}
$$

Equations (3) and (4) are discussed in previous section for deriving the probability of box having message at time $t$. Equation (5) gives the probability that collector node $i$ has the message at time $t$, which not only depends on previous state but also the chance of non-reception of message at any box. Thereby, the expected number of informed collectors at time $t$ can be obtained as $\mu_{t}=\sum_{i=1}^{N_{c}} p_{i, t}$.

Theorem 2 Given the non-zero limiting probability $q_{(i, b)}$ of any collector $i$ and $\alpha_{b, t}$ derived from Theorem 1 . For large $t, \mu_{t}=N_{c}$.

Proof By rearranging (5), we can get

$p_{i, t}=1-\left(1-p_{i, t-1}\right) \prod_{b=1}^{B}\left(1-\alpha_{b, t} q_{(i, b), t}\right)$

Let $r_{i, t}=\prod_{b=1}^{B}\left(1-\alpha_{b, t} q_{(i, b), t}\right)$ for short and iteratively solve the equation, then

$$
\begin{aligned}
p_{i, t}= & 1-\left(1-p_{i, t-1}\right) r_{i, t} \\
= & 1-r_{i, t}+p_{i, t-1} r_{i, t} \\
= & 1-r_{i, t}+\left(1-r_{i, t-1}+p_{i, t-2} r_{i, t-1}\right) r_{i, t} \\
= & \cdots \\
= & 1-r_{i, t}+\sum_{m=0}^{t}\left(\prod_{n=m+1}^{t} r_{i, n}\right)\left(1-r_{i, m}\right) \\
& \quad+\prod_{m=1}^{t} r_{i, m} p_{i, 0}
\end{aligned}
$$

For large $t$, we let $r_{i}$ to be the limiting probability of $r_{i, t}$ and $p_{i, 0}=0$, then

$p_{i, t}=\frac{1-r_{i}}{1-r_{i}}=1$

Thus, $\mu_{t}=\sum_{i=1}^{N_{c}} p_{i, t}=N_{c}$.

From Theorem 2, we can see that all message collectors will finally receive the message from the thrown box in network. However, when either $q_{(i, b), t}$ or $\alpha_{b, t}$ is always zero for any box $b$, the probability $p_{i, t}$ will be zero and the collector $i$ will never collect any messages. The above conditions denote the case that the message sender and collector have no common visited boxes according to their mobility patterns. In order to make them communicate, some boxes have to be deployed in their common active areas.

\section{Cooperative collectors}

A cooperative collector can be viewed as the sender without having data in the beginning and starts to relay data when receiving message from box. The model for cooperative collectors subsume three similar equations in previous 
section, where the only distinct equation about probability $\beta_{b, t}$ is given as follows.

$\beta_{b, t}=\prod_{j=1}^{N_{s}}\left(1-m_{(j, b), t}\right) \prod_{j=1}^{N_{c}}\left(p_{j, t-1}\left(1-q_{(j, b), t}\right)+1-p_{j, t-1}\right)$

Compared with (3), the impact on $\beta_{b, t}$ from the cooperative collectors comes in the form of the second part of product. Since every cooperative collector may behave like the sender and scatters message at certain time, the probability $\beta_{b, t}$ also depends on the states of collectors. If a collector does not hold any message at previous time $t-1$ or it has the message without reaching box $b$ at time $t$, then this collector will contribute to the probability $\beta_{b, t}$. In order to derive $\mu_{t}$, the second product term on the right of (6) can be approximated as constant coefficient. Then based on the result from Theorem 2, $\mu_{t}$ will approach to $N_{c}$ for large $t$.

\section{Latency of message collection}

We concentrate on the latency between when a collector starts to move and it receives the data for the first time. In this latency model, as we give the assumption in Sect. 3, the transition time between spots is relatively short compared with pause time at boxes, so the transition time is ignored when we consider the latency. Moreover, we focus on the message collection with independent collectors. In other words, once a collector retrieves the data, it finishes its task and will not relay message to other collectors.

Before the collector starts to gather message from the thrown boxes, the senders could already spread messages in network. So we make use of the limiting probability of $\alpha_{b}$ to approximate the message distribution at the boxes. For large $t$, the limiting probability for box $b$ is used to derive the latency and its expression from Theorem 1 is shown as follows:

$\alpha_{b}=\frac{1-\prod_{j=1}^{N_{s}}\left(1-m_{(j, b)}\right)}{1-\prod_{j=1}^{N_{s}}\left(1-m_{(j, b)}\right)+e}$

After that, we construct a Markov Chain to model the message collection for any collector. A sample Markov Chain with four states is shown in Fig. 2. Box $i$ and Box $j$ denote the states without message stored and their corresponding absorption states expressed by the red circles are the states of box having valid message. The expression on each directed arc denotes the transition probability of mobile collector among these states. Once a collector enters into either state $i_{m}$ or $j_{m}$, it means the collector retrieves the data

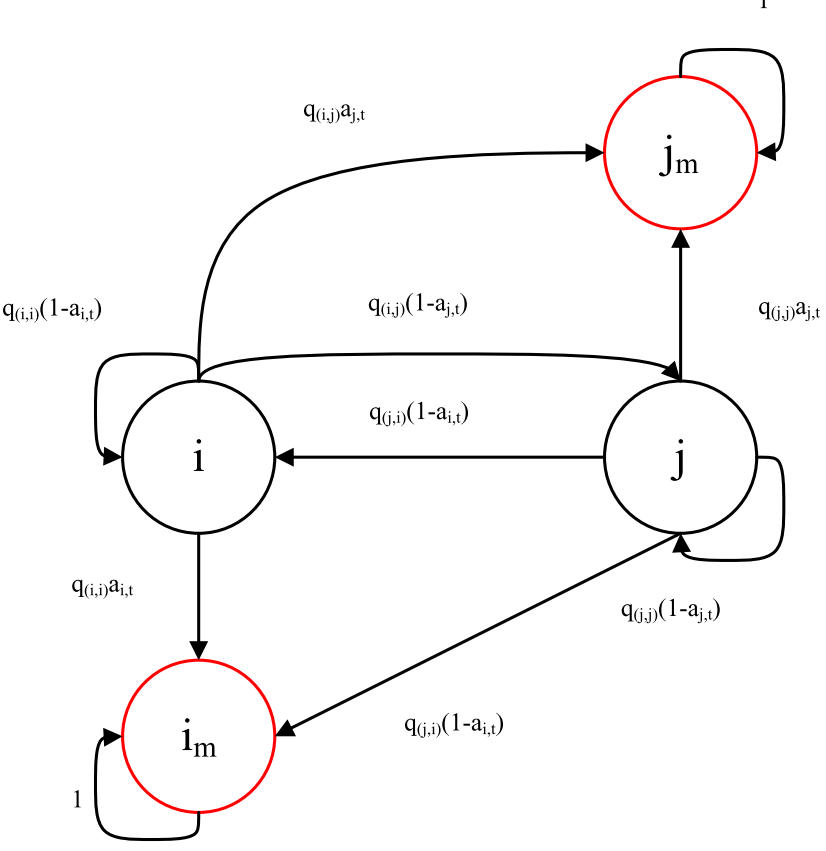

Fig. 2 Mobility matrix based Markov Chain

and stops. Thereby, each box has an extra state to indicate the message reception and there are total $2 \times B$ states in the chain.

Theorem 3 Given above Markov Chain model, limiting probability vector $\alpha=\left[\alpha_{1}, \ldots, \alpha_{B}\right]^{\prime}$ and mobility matrix $Q_{i}$ for collector $i$, then $h_{i, t}=A_{i, 0}\left(\prod_{k=1}^{t-1} Q_{i} \bar{A}\right)\left(Q_{i} A\right) \mathbf{1}$ where $A=\operatorname{diag}(\alpha), \bar{A}=I-A$ and $A_{i, 0}$ is a vector representing the starting box from which collector $i$ moves.

Proof The transition matrix $R$ of the Markov model for collector $i$ is provided first. The set $\{1, \ldots, B\}$ represents the states of box without message and the set $\left\{1_{m}, \ldots, B_{m}\right\}$ denotes the states of box having message. Then entry $r_{i j}$ in matrix $R$ is

$$
\begin{array}{ll}
r_{(i, j)}=q_{(i, j)}\left(1-\alpha_{j}\right) & \text { if } i, j \in[1, B] \\
r_{(i, j)}=q_{(i, j)} \alpha_{j} & \text { if } i \in[1, B], j \in\left[1_{m}, B_{m}\right] \\
r_{(i, i)}=1 & \text { if } i \in\left[1_{m}, B_{m}\right] \\
r_{(i, j)}=0 & \text { else }
\end{array}
$$

Then, the general form of matrix $R$ can be shown as follows

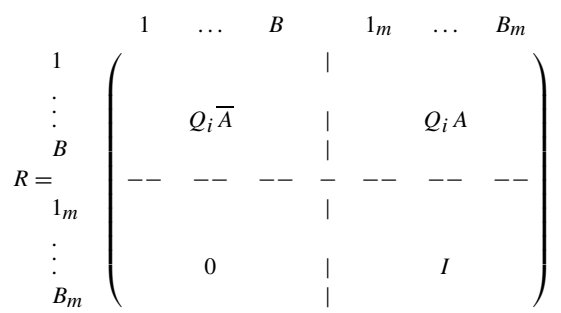


Based on Phase-type distribution, the probability that the message can be collected by collector $i$ at time $t$ for the first time is

$h_{i, t}=A_{i, 0}\left(\prod_{k=1}^{t-1} Q_{i} \bar{A}\right)\left(Q_{i} A\right) \mathbf{1}$

$A_{i, 0}$ is the initial location vector.

From Theorem 3, we know that the latency will decrease as the probability $\alpha_{b}$ increases. When $e=1$, the message will only stored at the box where the message senders stay and the resultant latency denotes the first contact time with message sender. After that, the expected latency for collector $i$ is $\sum_{t} t h_{i, t}$ and the expected number of informed collectors by time $t, \eta_{t}=\sum_{i=1}^{N_{c}} \sum_{j=1}^{t} h_{i, j}$.

\section{Numerical result}

In this section, several numerical results are given to demonstrate the features of thrown box based message dissemination and to validate our model. We mainly capitalize on three different types of network graph to generate various mobility matrices for illustration, including complete graph, cluster based topology and linear topology. The vertex in the network graph denotes a box deployed at certain location and the edge represents the path between any two boxes. The network simulator ONE [15] is employed to validate the analytical model.

\subsection{Mobility model}

Based on the underlying network topology, the mobility of each node is expressed as a stochastic matrix where the nonzero value denotes the probability of choosing certain path at next time step. In the first complete graph based scenario, any pair of boxes has a path between them and the transition probabilities from certain box to other boxes are randomly chosen. The sample mobility matrix with only six boxes is given as below.

$$
\left(\begin{array}{cccccc}
0.31 & 0.33 & 0.08 & 0.22 & 0.04 & 0.02 \\
0.2 & 0.1 & 0.11 & 0.12 & 0.19 & 0.28 \\
0.05 & 0.29 & 0.26 & 0.07 & 0.21 & 0.12 \\
0.13 & 0.2 & 0.04 & 0.22 & 0.22 & 0.19 \\
0.05 & 0.01 & 0.19 & 0.26 & 0.24 & 0.25 \\
0.06 & 0.07 & 0.13 & 0.29 & 0.25 & 0.2
\end{array}\right)
$$

In order to simulate localized mobility, we provide the cluster based scenario where mobile nodes have higher probability to visit several local boxes and lower chance to move out of them. The corresponding probability reflects the visiting preference when making the movement decision. The following matrix gives an example to represent the cluster based mobility.

$\left(\begin{array}{cccccc}0.13 & 0.35 & 0.14 & 0 & 0.38 & 0 \\ 0.6 & 0.4 & 0 & 0 & 0 & 0 \\ 0.33 & 0 & 0.03 & 0.6 & 0.04 & 0 \\ 0 & 0 & 0.65 & 0.35 & 0 & 0 \\ 0.22 & 0 & 0.27 & 0 & 0.44 & 0.07 \\ 0 & 0 & 0 & 0 & 0.8 & 0.2\end{array}\right)$

At last, linear topology presents a more restricted mobility where the node at non-boundary boxes only have three movement options: stay, moving forward and moving backward. A sample matrix is shown below.

$$
\left(\begin{array}{cccccc}
0.02 & 0.98 & 0 & 0 & 0 & 0 \\
0.32 & 0.12 & 0.56 & 0 & 0 & 0 \\
0 & 0.33 & 0.24 & 0.43 & 0 & 0 \\
0 & 0 & 0.40 & 0.06 & 0.54 & 0 \\
0 & 0 & 0 & 0.34 & 0.36 & 0.29 \\
0 & 0 & 0 & 0 & 0.46 & 0.54
\end{array}\right)
$$

In the following sections, there are total 20 boxes arranged under these three network scenarios. The mobility matrices are generated first and then the movement trace of mobile node is derived accordingly. According to our network assumption, the transition time between boxes is ignored. At each time step, every mobile node will stay at certain box and choose the next box based on its stochastic matrix.

\subsection{Process of message dissemination among boxes}

In this section, the numerical results based on the analytical model are given and then the simulation is introduced for model validation. Firstly, the impact of probability of message expiration on the number of message boxes is studied under different network scenarios. There are total 20 thrown boxes and 2 message senders in numerical results. From the results shown in Fig. 3, there exists the limiting number of message boxes. When the probability of message expiration is zero, the maximum number of message boxes will eventually reach to 20 . As the expiration probability increases, the number of message boxes reduces and the limiting amount of message box is less than 20 . When the probability becomes 1 , only the boxes where message senders stay have message stored and thereby the amount is less or equal than 2. Furthermore, various network scenarios lead to different latencies for reaching stable state. The sending process costs the shortest time in the network with complete graph and the longest time in linear based network. Also from the curves in the bottom of 


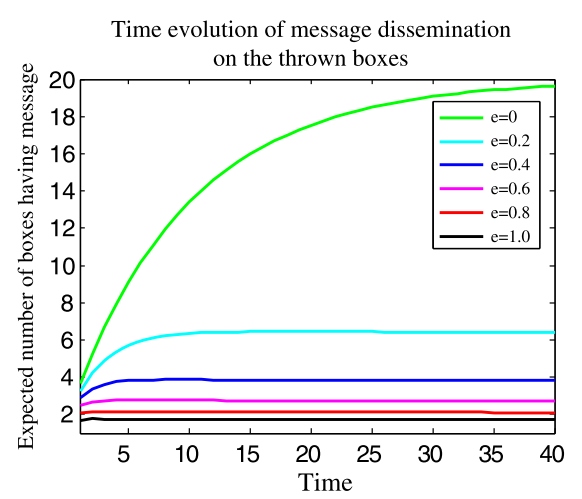

(a) Complete graph based scenario

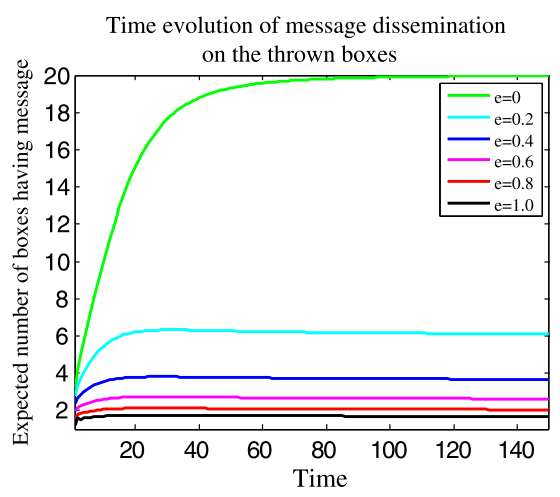

(b) Cluster based scenario

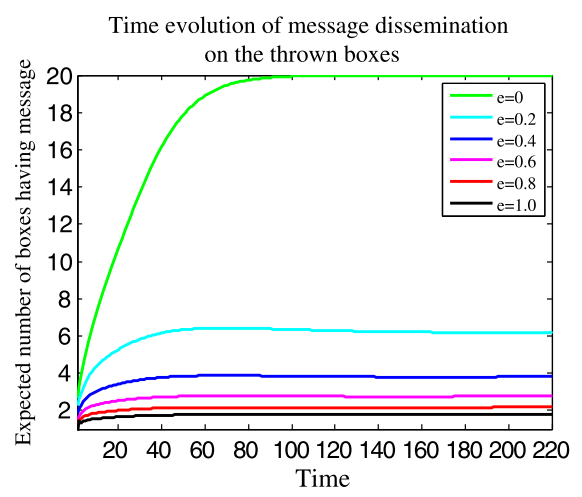

(c) Linear topology cenario

Fig. 3 Time evolution of thrown boxes having message under different scenarios

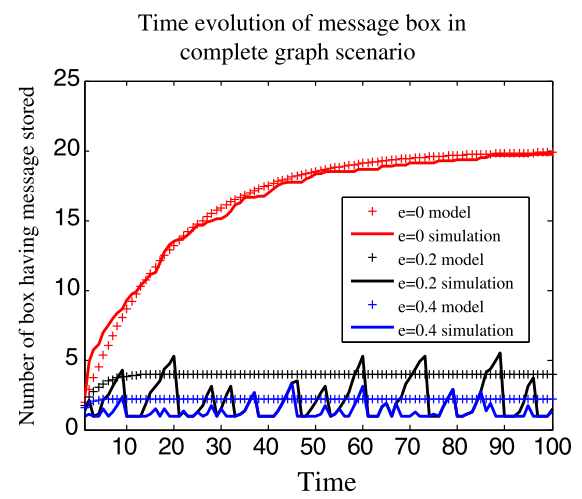

(a) Complete graph based scenario

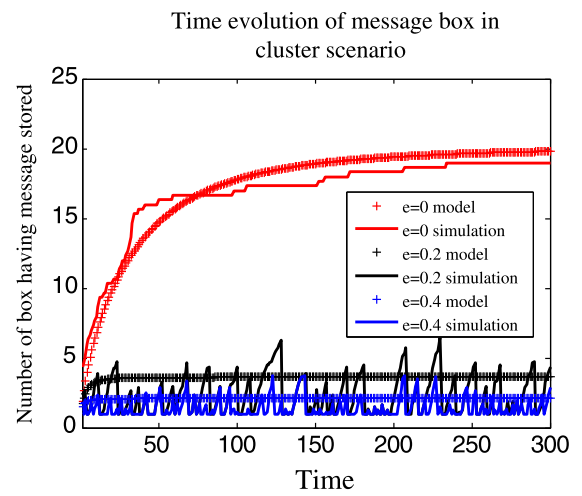

(b) Cluster based scenario

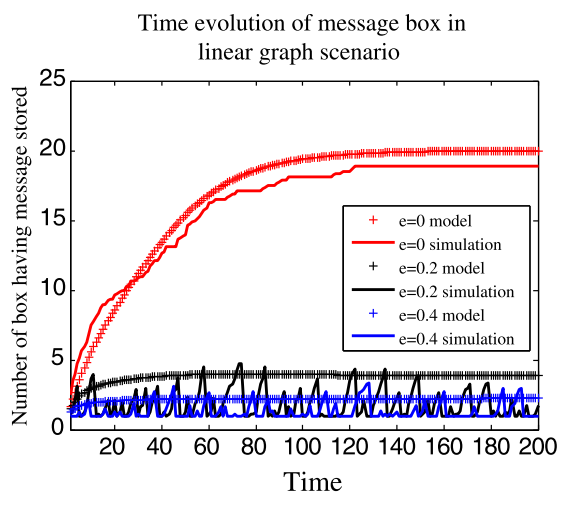

(c) Linear topology scenario

Fig. 4 Comparison between model and simulation in different scenarios

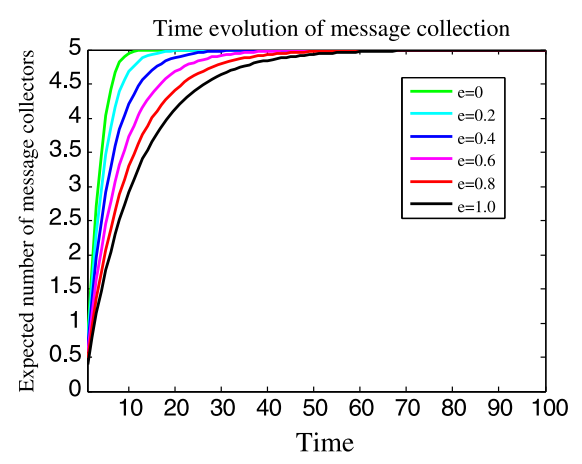

(a) Complete graph based scenario

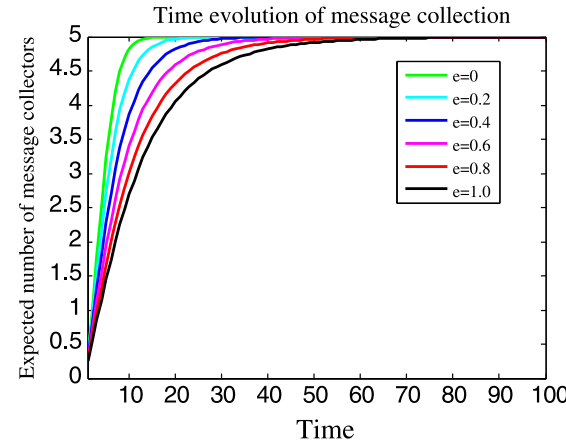

(b) Cluster based scenario

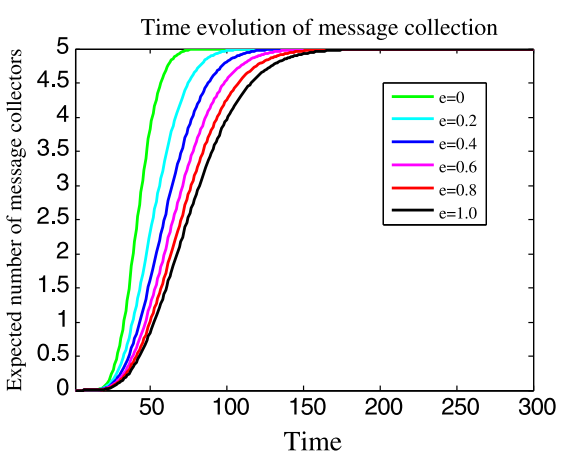

(c) Linear topology scenario

Fig. 5 Time evolution of informed collectors in different scenarios

each figure, we find that the expected numbers of boxes are close but the expiration probability varies a lot. This feature may help design efficient buffer management at the thrown box.

Secondly, the comparison between model and simulation is given in Fig. 4. There are 20 thrown boxes in network but only one sender is involved. Using different net- work scenarios and various message expiration probabilities, the averaged simulation results approximately agree with the results of analytical model. The difference may result from the average error and message transmission delay. The wave-like curves show the evolution of message box in the simulation while the model curves are smooth. 


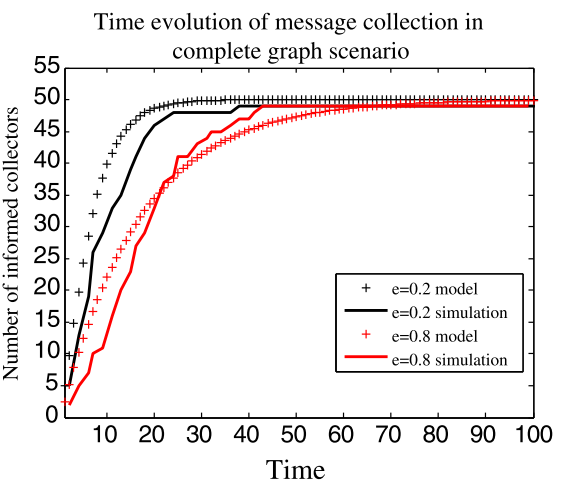

(a) Complete graph based scenario

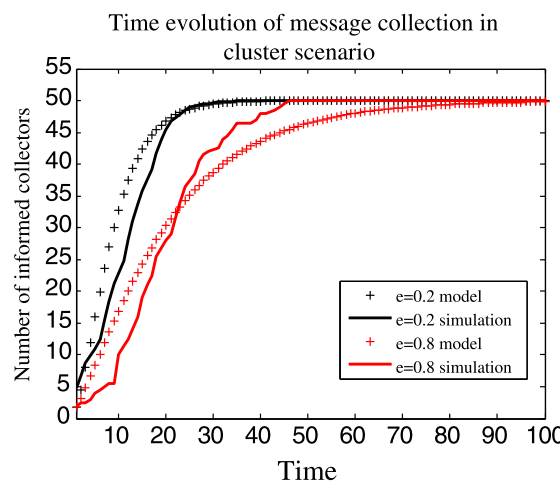

(b) Cluster based scenario

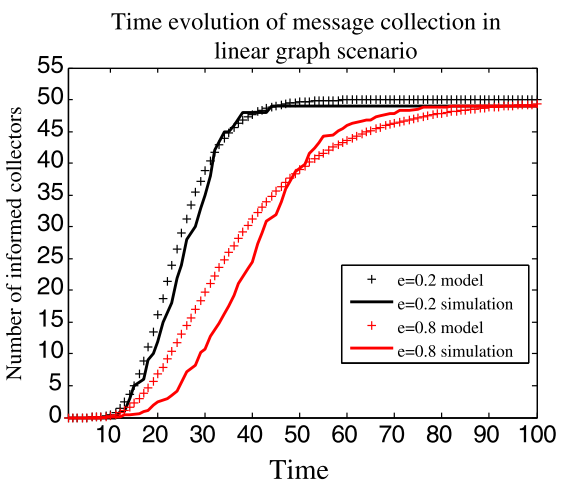

(c) Linear topology scenario

Fig. 6 Comparison between model and simulation in different scenarios

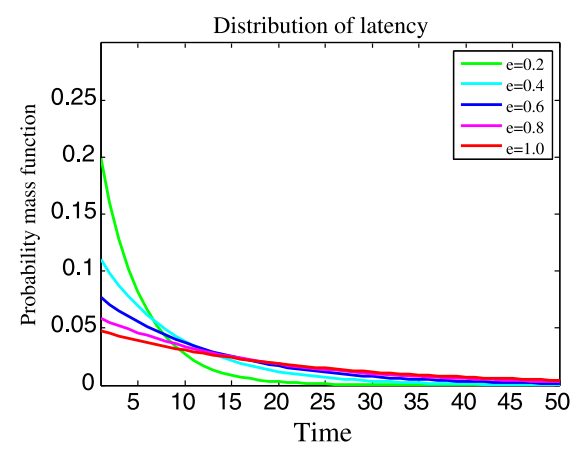

(a) Complete graph based scenario

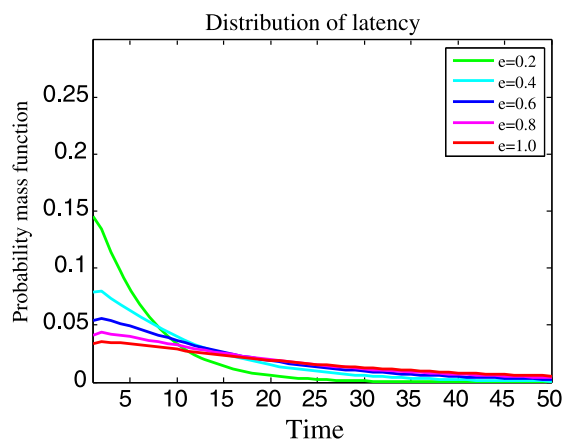

(b) Cluster based scenario

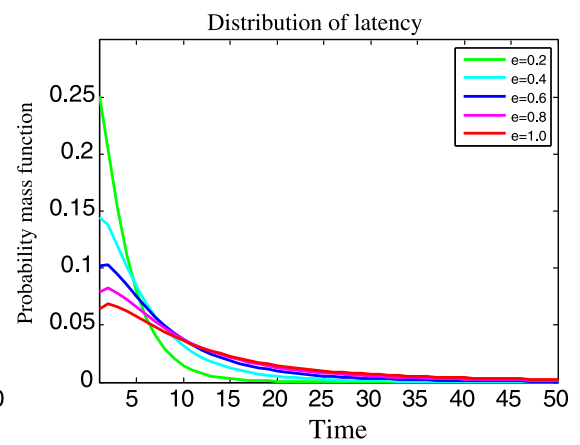

(c) Linear topology scenario

Fig. 7 Latency distribution with various expiration probabilities in different scenarios

\subsection{Process of message collection}

The time evolution of message collection is shown by deriving the expected number of cooperative message collectors against time. Various probabilities of message expiration and 5 message collectors are employed to demonstrate the features of analytical model on message collection. The resultant curves show that the latency becomes longer as expiration probability increases. In addition, the curves in Fig. 5a and Fig. 5b are similar but varies in small delay. In Fig. 5c, we can observe that the collectors need a longer time to retrieve data. And there is no collector having message in the beginning due to the initial far distance between collector and sender.

After that, the simulation results are given in Fig. 6. The simulation setting includes one message sender, 20 thrown boxes and 50 message collectors. The message sender and message collectors have different mobility models and starting points. The curves in Fig. 6 indicate that the analytical model approximately matches the simulation results under different scenarios. The average error and transmission delay cause the difference betweens simulation and model.

\subsection{Distribution of latency}

Given the same network scenarios, the latency distribution about when the mobile collector receives the message for the first time is studied as well. One collector and one sender are used to derive the latency in the network with total 20 boxes. From Fig. 7, we find that the expected latency in linear topology scenario is the smallest. That is because in such scenario the messages exist with higher probability at several boxes which are likely visited by the collector. For the other cases, the collector needs the longest expected latency in the cluster based scenario and the averaged latency for complete graph based scenario is in-between. For each subfigure, as the expiration probability increases, the expected latency grows accordingly.

\section{Conclusion}

In this paper, we study the thrown-box assisted message dissemination and propose the corresponding models to analyze the message delivery rate and related latency distribution. Differing in the phases of message passing, we first model the message dissemination among boxes and derive 
the time evolution relating the number of the boxes containing messages. Then, we investigate the process of message collection. We calculate the expected number of informed collectors. Further, the latency distribution of message collection is derived based on a discrete Markov Chain model. We have some interesting numerical results for three different network topologies. The results will help buffer management and delay management in delay tolerant networks.

\section{References}

1. Balasubramanian, A., Levine, B., \& Venkataramani, A. (2007). DTN routing as a resource allocation problem. Computer Communication Review, 37, 373-384.

2. Bales, K., French, J. A., \& Dietz, J. M. (2002). Explaining variation in maternal care in a cooperatively breeding mammal. Animal Behaviour, 63, 453-461.

3. Beni, G., \& Wang, J. (1989). Swarm intelligence in cellular robotic systems. In Proceed. NATO advanced workshop on robots and biological systems.

4. Caro, G. D., \& Dorigo, M. (1998). Antnet: distributed stigmergetic control for communications networks. The Journal of Artificial Intelligence Research, 9, 317-365.

5. Cronin, K. A., Kurian, A. V., \& Snowdon, C. T. (2005). Cooperative problem solving in a cooperatively breeding primate. Animal Behaviour, 69, 133-142.

6. Daly, E., \& Haahr, M. (2007). Social network analysis for routing in disconnected delay-tolerant MANETs. In Proc. MobiHoc 07.

7. Das, S., \& Gerla, M. (2004). SPAWN: a swarming protocol for vehicular ad hoc networks. In The 1st ACM VANET, Oct.

8. Detrain, C., \& Deneubourg, J.-L. (2002). Complexity of environment and parsimony of decision rules in insect societies. Biological Bulletin, 202, 268-274.

9. Drea, C. M. (1998). Social context affects how rhesus monkeys explore their environment. American Journal of Primatology Supplement, 44, 205-214.

10. Drea, C. M. (2006). Studying primate learning in group contexts: tests of social foraging, response to novelty, and cooperative problem solving. Methods, 38, 162-177.

11. Gerla, M., Yi, Y., Xu, K., \& Hong, X. (2003). Team communications among airborne swarms. In 2003 IEEE aerospace conference, March.

12. Ghosh, J., Ngo, H. Q., Yoon, S., \& Qiao, C. (2007). On a routing problem within probabilistic graphs and its application to intermittently connected networks. In IEEE INFOCOM.

13. Hui, P., Crowcroft, J., \& Yoneki, E. (2008). Bubble rap: socialbased forwarding in delay tolerant networks. In Proceedings of the 9th ACM international symposium on mobile ad hoc networking and computing (pp. 241-250).

14. Jain, S., Fall, K., \& Patra, R. (2004). Routing in a delay tolerant network. In Proc. ACM Sigcomm (pp. 145-158).

15. Keränen, A., Ott, J., \& Kärkkäinen, T. (2009). The ONE simulator for DTN protocol evaluation. In SIMUTools '09: proceedings of the 2nd international conference on simulation tools and techniques, New York, NY, USA, ICST.

16. Lewis, R. J. (2006). Scent marking in sifaka: no one function explains it all. American Journal of Primatology, 6(68), 622-636.

17. Rajagopalan, S., \& Shen, C.-C. (2005). ANSI: a unicast routing protocol for mobile ad hoc networks using swarm intelligence. In ICAI 2005: international multiconference in computer science and computer engineering, special session on swarm intelligence, June.
18. Sarafijanovic-Djukic, N., Piorkowski, M., \& Grossglauser, M. (2006). Island hopping: efficient mobility-assisted forwarding in partitioned networks. In SECON'06 (Vol. 1, pp. 226-235).

19. Selvakennedy, S., Sinnappan, S., \& Shang, Y. (2007). A biologically-inspired clustering protocol for wireless sensor networks. Computer Communications, 30, 2786-2801.

20. Shen, C.-C., \& Jaikaeo, C. (2005). Ad hoc multicast routing algorithm with swarm intelligence. ACM Mobile Networks and Applications Journal, 10, 47-59.

21. Shin, M., Hong, S., \& Rhee, I. (2008). DTN routing strategies using optimal search patterns. In CHANTS'08.

22. Smuts, B. B., Cheney, D. L., \& Seyfarth, R. M. (1987). Primate societies. Chicago: The University of Chicago Press.

23. Spyropoulos, T., Psounis, K., \& Raghavendra, C. S. (2006). Performance analysis of mobility-assisted routing. In MobiHoc 06: proceedings of the seventh ACM international symposium on mobile ad hoc networking and computing (pp. 49-60).

24. Spyropoulos, T., Psounis, K., \& Raghavendra, C. S. (2008). Efficient routing in intermittently connected mobile networks: the multiple-copy case. IEEE/ACM Transaction on Networking, 16. doi:10.1109/TNET.2007.897964.

25. Suzuki, J., \& Suda, T. (2005). A middleware platform for a biologically inspired network architecture supporting autonomous and adaptive applications. IEEE Journal on Selected Areas in Communications, 23(2), 249-260.

26. Viswanathan, G. M., Afanasyev, V., Buldyrev, S. V., Murphy, E. J., Prince, P. A., \& Stanley, H. E. (1996). Levy flights search patterns of wandering albatrosses. Nature, 381, 413-415.

27. Wei-jen Hsu, K. P., Spyropoulos, T., \& Helmy, A. (2007). Modeling time-variant user mobility in wireless mobile networks. In IEEE INFOCOM.

28. Zhao, W., Ammar, M., \& Zegura, E. (2004). A message ferrying approach for data delivery in sparse mobile ad hoc networks. In Proceedings of the 5th ACM international symposium on mobile ad hoc networking and computing (pp. 187-198).

29. Zhao, W., Chen, Y., Ammar, M., Corner, M., Levine, B., \& Zegura, E. (2006). Capacity enhancement using throwboxes in dtns. In Proc. IEEE intl conf on mobile ad hoc and sensor systems (MASS) (pp. 31-40).

30. Zheng, Q., Hong, X., Wang, P., Tang, L., \& Liu, J. (2008). Delay management in delay tolerant networks. International journal of network Management, special issue: mathematical methods in network management.

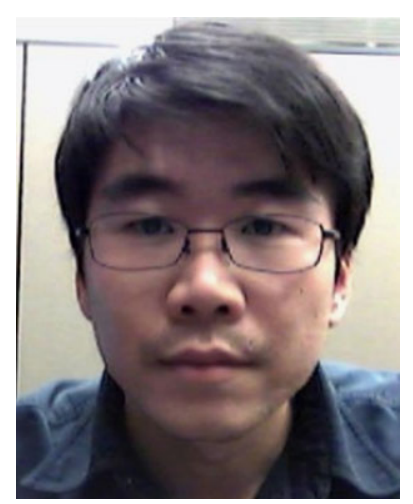

Bo Gu is a Ph.D. student in the department of computer science at the University of Alabama. He received M.S. degree in computer science from the University of Alabama at 2009 and M.S. degree in computer science from Beihang University, P.R. China at 2007. His research interests include wireless networking, mobile networking, delay tolerant network and network modeling. 


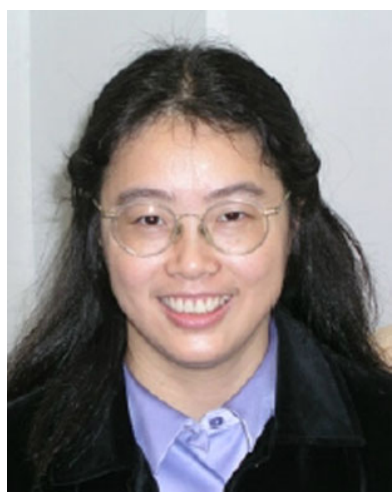

Xiaoyan Hong is an associate professor in the Department of Computer Science at the University of Alabama, USA. She received her $\mathrm{Ph} . \mathrm{D}$. degree in Computer Science from the University of California, Los Angeles in 2003. Her research interests include mobile and wireless networks, future wireless Internet, vehicle networks and mobility modeling.

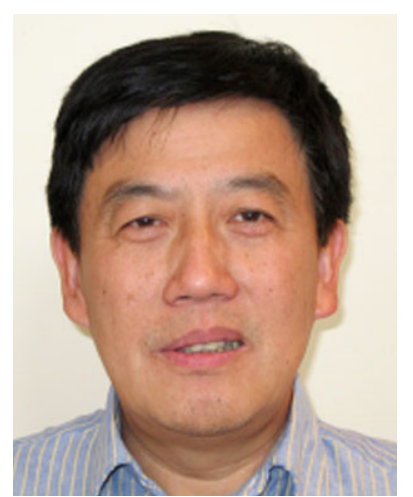

Pu Wang received his M.S. and Ph.D. degrees from Lehigh University, Bethlehem, PA respectively in 1985 and 1990 . He is currently a professor with the Department of Mathematics of University of Alabama. His primary research interests are theory and applications of stochastic processes. Some of the applications involved in his research include queueing theory and queueing networks, telecommunications networks, and stochastic transport models in groundwater. 